Supporting Information

\title{
Molecular Simulation Study on Factors Affecting Carbon Dioxide Adsorption on Amorphous Silica Surfaces
}

\author{
Ki Chul Kim ${ }^{1,2, *}$ and Seung Soon Jang ${ }^{1,3,4, *}$ \\ 1 Computational NanoBio Technology Laboratory, School of Materials Science and \\ Engineering, Georgia Institute of Technology, 771 Ferst Drive NW, Atlanta, Georgia \\ 30332-0245, USA \\ 2 Division of Chemical Engineering, Konkuk University, Seoul 05029, The Republic of \\ Korea \\ 3 Institute for Electronics and Nanotechnology, Georgia Institute of Technology, Atlanta, \\ GA 30332, USA \\ 4 Parker H. Petit Institute for Bioengineering and Bioscience, Georgia Institute of \\ Technology, Atlanta, GA 30332, USA
}

\section{Corresponding Authors}

*Emails: kich2018@konkuk.ac.kr (K. C. Kim); seungsoon.jang@mse.gatech.edu (S. S. Jang) 


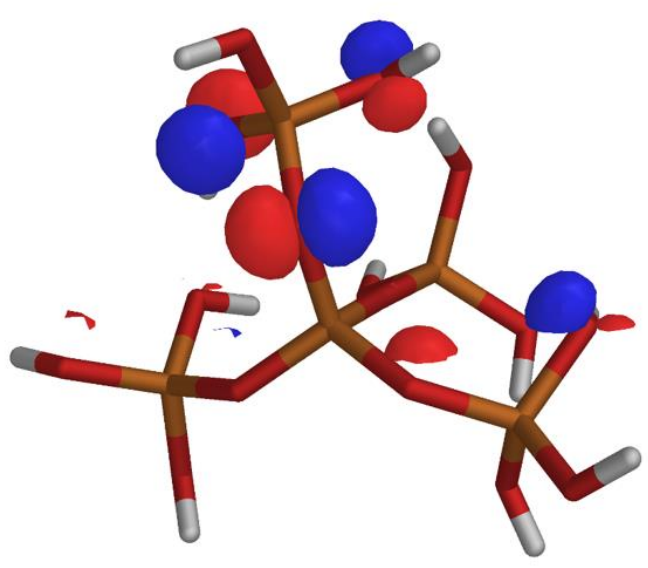

HOMO

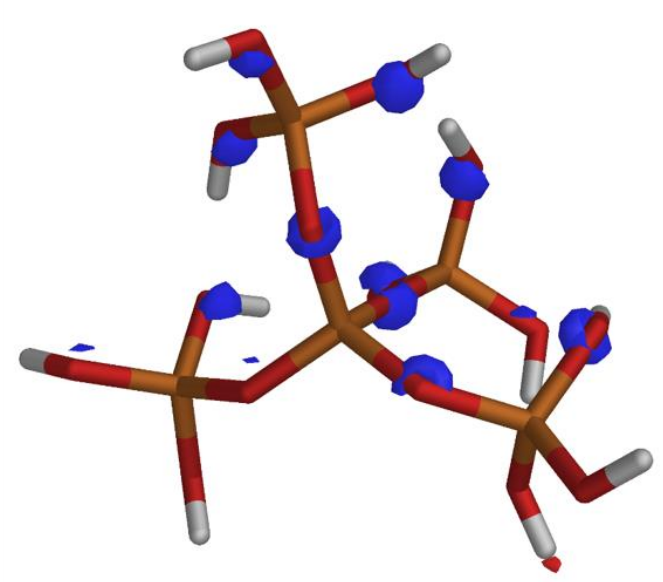

LUMO

Figure S1. The cluster model $\left(\mathrm{Si}\left(\mathrm{OSi}(\mathrm{OH})_{3}\right)_{4}\right)$ employed to predict atomic charges of $\mathrm{Si}, \mathrm{O}$, and $\mathrm{H}$ for an amorphous silica model without any $\mathrm{Si}-\mathrm{H}$ defects. The atoms with orange, red, and white in color depict silicon, oxygen, and hydrogen, respectively.

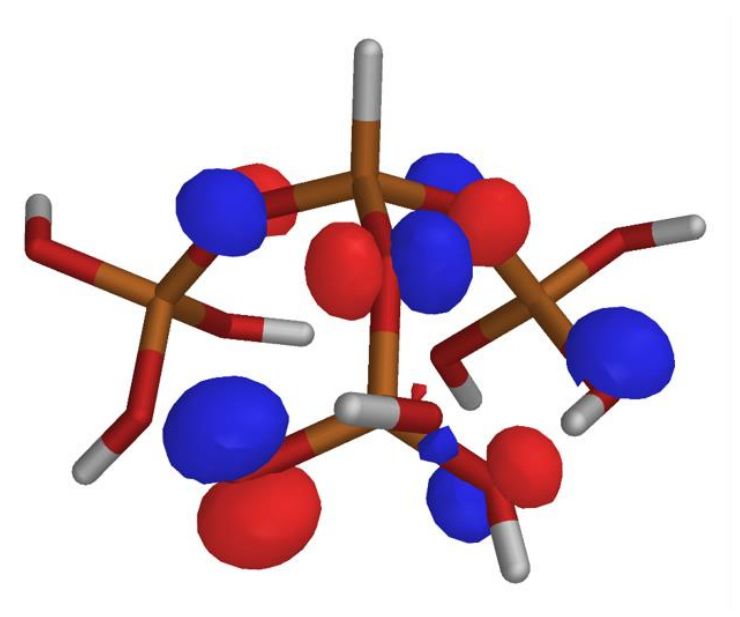

HOMO

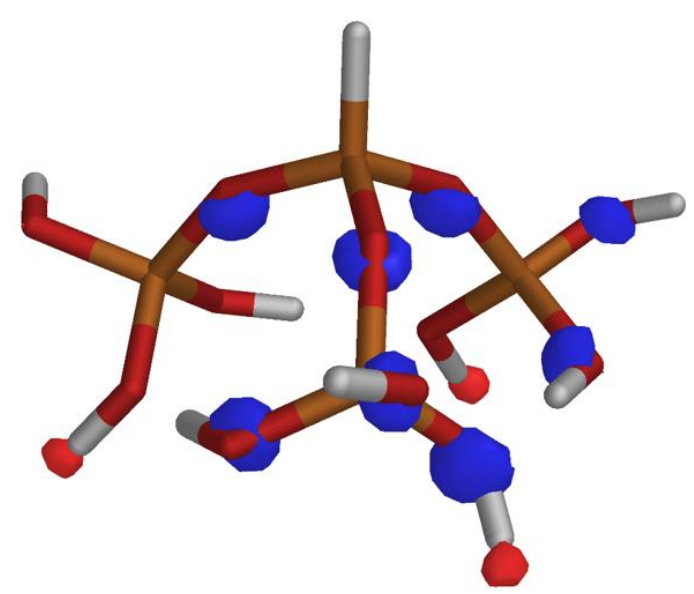

LUMO

Figure S2. The cluster model $\left(\mathrm{Si}\left(\mathrm{OSi}(\mathrm{OH})_{3}\right)_{3} \mathrm{H}\right)$ employed to predict atomic charges of $\mathrm{Si}$, O, and $\mathrm{H}$ for a tetrahedral geometry of $\mathrm{Si}$ with one $\mathrm{Si}-\mathrm{H}$ defect. The atoms with orange, red, and white in color depict silicon, oxygen, and hydrogen, respectively. 


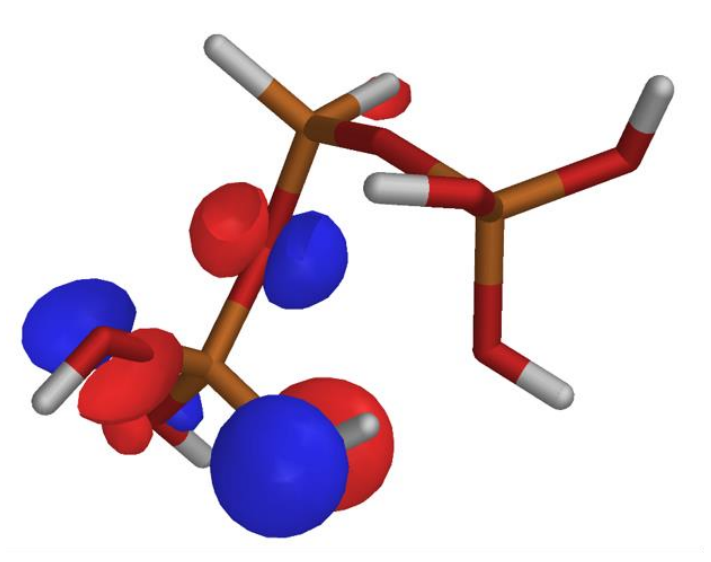

HOMO

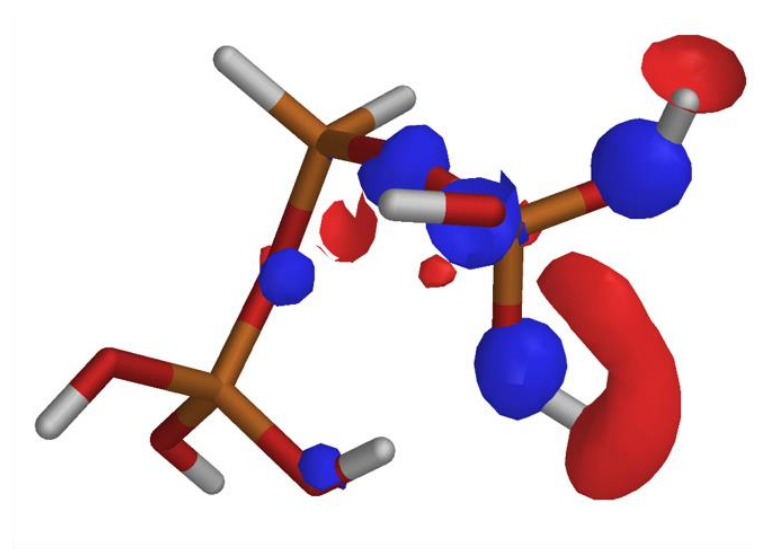

LUMO

Figure $\mathrm{S}_{3}$. The cluster model $\left(\mathrm{Si}\left(\mathrm{OSi}(\mathrm{OH})_{3}\right)_{2} \mathrm{H}_{2}\right)$ employed to predict atomic charges of $\mathrm{Si}, \mathrm{O}$, and $\mathrm{H}$ for a tetrahedral geometry of $\mathrm{Si}$ with two $\mathrm{Si}-\mathrm{H}$ defects. The atoms with orange, red, and white in color depict silicon, oxygen, and hydrogen, respectively.

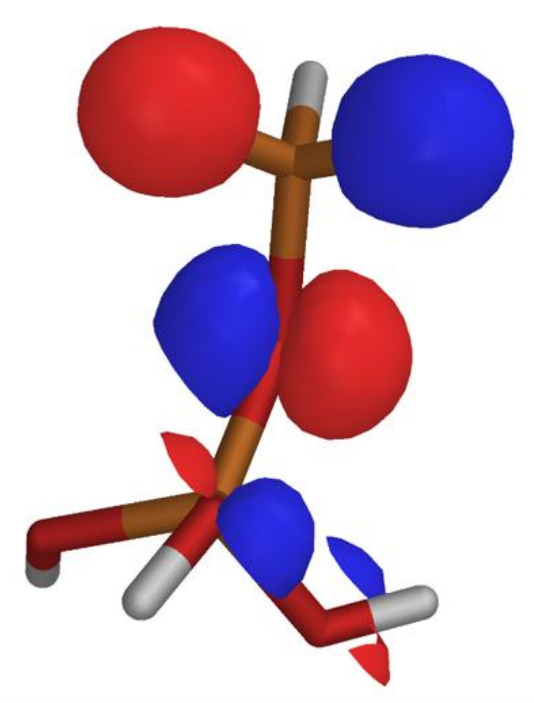

HOMO

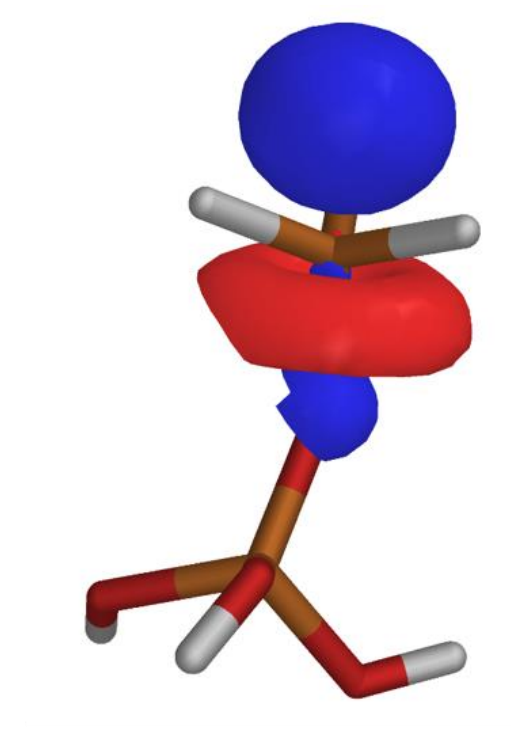

LUMO

Figure $\mathrm{S}_{4}$. The cluster model $\left(\mathrm{Si}\left(\mathrm{OSi}(\mathrm{OH})_{3}\right) \mathrm{H}_{3}\right)$ employed to predict atomic charges of $\mathrm{Si}, \mathrm{O}$, and $\mathrm{H}$ for a tetrahedral geometry of $\mathrm{Si}$ with three $\mathrm{Si}-\mathrm{H}$ defects. The atoms with orange, red, and white in color depict silicon, oxygen, and hydrogen, respectively. 
Table S1. A full list of the DFT-predicted atomic charges for the cluster models with varied numbers of $\mathrm{Si}-\mathrm{H}$ defects (see Figures $\mathrm{S}_{1}-\mathrm{S}_{4}$ ).

\begin{tabular}{lllll}
\hline Si: tetrahedral geometry & \multicolumn{3}{c}{ Atom type } \\
& Si & $\mathbf{O}$ & H of O-H & H of Si-H \\
\hline Without Si-H defect & 1.08 & -0.60 & 0.33 & - \\
With one Si-H defect & 1.06 & -0.60 & 0.33 & -0.13 \\
With two Si-H defects & 0.91 & -0.60 & 0.33 & -0.11 \\
With three Si-H defects & 0.67 & -0.60 & 0.33 & \\
\hline
\end{tabular}

